\title{
BIBLIOTHEKSPRAKTIKUM WÄHREND DER \\ COVID-19-PANDEMIE: ERFAHRUNGEN MIT ONLINE-PRAKTIKA AN DER UNIVERSITÄTSBIBLIOTHEK WIEN
}

\section{von Tereza Kalová}

Zusammenfassung: Studierende der Bibliothekswissenschaft müssen auch in der jetzigen Situation unter häufig erschwerten Bedingungen berufliche Erfahrung in Bibliotheken sammeln. Bibliotheken sollten wiederum den wichtigen Input und neue Ideen, die Praktikant*innen liefern können, wegen der erhöhten Sicherheitsmaßnahmen nicht verlieren. In der Abteilung Repositorienmanagement PHAIDRA-Services der Universität Wien, die sich generell über ein großes Interesse von Praktikant*innen freut, wurde daher im Sommer 2020 ein neues Konzept für Online-Praktika entwickelt. Die erste Gruppe von vier Studierenden von der Humboldt-Universität zu Berlin hat im Oktober-November 2020 ein vierwöchiges Praktikum im Homeoffice absolviert. Im vorliegenden Beitrag wird aufdas Konzept des virtuellen Praktikums eingegangen. Weiters werden die Erfahrungen der beteiligten Mitarbeiter*innen der UB Wien und die Rückmeldungen der Praktikant*innen dargestellt. Die gesammelten Erfahrungen der Abteilung PHAIDRA zielen darauf ab, anderen Bibliotheken Ideen für eine mögliche Umstellung auf Online-Praktika zu bieten.

Schlagwörter: Universitätsbibliothek Wien; Bibliothekspraktikum; virtuelles Praktikum; COVID-19; Pandemie

\section{LIBRARY INTERNSHIP DURING THE COVID-19 PANDEMIC: EXPERIENCES WITH VIRTUAL INTERNSHIPS AT THE VIENNA UNIVERSITY LIBRARY}

\begin{abstract}
Even in the current situation, students of library science must gain professional experience in libraries under often difficult conditions. Libraries, in turn, should not lose the important input and new ideas that interns can provide because of the increased security measures. Many students choose to complete a part of their compulsory internship in the department of Repository Management PHAIDRA-Services of the University of Vienna every year. To continue this tradition during the crisis, a new concept for online internships was developed in the summer of 2020. The first group of four students from the Humboldt University of Berlin completed a four-week remote internship in October-November 2020. The paper discusses the concept for the virtual internship, as well as the feedback from both colleagues and interns. Lessons learned are presented in order to help other libraries that are considering the shift to virtual internships.
\end{abstract}


Keywords: Vienna University Library; library internship; remote internship; COVID-19; Pandemic

DOI: https://doi.org/10.31263/voebm.v73i3-4.5371

(C) Tereza Kalová

(c) (i) Dieses Werk ist - exkl. einzelner Logos und Abbildungen - lizenziert unter

Sammeln praktischer beruflicher Erfahrung in Form von Praktika gehört besonders im D-A-CH-Bereich zu der bibliothekarischen Ausbildung dazu. Ein Praktikum kann dabei helfen, theoretisches Wissen aus dem Studium in der Praxis zu erproben und ermöglicht die Knüpfung wichtiger Kontakte.

Was bleibt aber übrig, wenn typische Inhalte eines Bibliothekspraktikums wie eine Führung durch die Lesesäle oder historische Sammlungen aufgrund erhöhter Sicherheitsmaßnahmen und fehlenden Personals vor Ort entfallen? Die derzeitige Situation hat uns in der Abteilung Repositorienmanagement PHAIDRA-Services der UB Wien dazu veranlasst, das Format eines Online-Praktikums auszuprobieren und ein rein virtuelles Praktikum anzubieten.

Im vorliegenden Text werden Reaktionen von bibliothekswissenschaftlichen Studiengängen auf die Pandemie bezüglich Praktikumsvorgaben thematisiert, das Konzept für virtuelle Praktika präsentiert, sowie die Erfahrungen der Betreuer*innen und das Feedback der ersten Praktikant*innen vom Oktober-November 2020 dargestellt.

\section{Einfluss der Pandemie auf Praktikumsvorgaben}

Um den möglichen Pandemie-bedingten Änderungen der Praktikumsvorgaben in bibliothekswissenschaftlichen Studiengängen nachzugehen, wurde im November 2020 eine kurze formlose Umfrage durchgeführt.

Hierbei wurden bei 14 deutschen und österreichischen Einrichtungen, die bibliotheks- und informationswissenschaftliche Lehr- und Studiengänge anbieten, die folgenden Fragen gestellt:

- Hat die Pandemie einen Einfluss auf die Vorgaben für Praktika in Ihren Lehr- und Studiengängen gehabt?

- Was hat sich eventuell geändert? 
An dieser Stelle muss angemerkt werden, dass sich die konkreten Vorgaben insbesondere bezüglich Umfang in den verschiedenen Lehr-, Bachelor- und Masterstudiengängen stark voneinander unterscheiden. Auf diese Unterschiede im Detail einzugehen, würde den Rahmen dieses Artikels sprengen. Demnach werden in den folgenden Absätzen die möglichen Änderungen der Praktikumsvorgaben unabhängig von den vorbestehenden Vorgaben an den einzelnen Einrichtungen beschrieben. Das Ziel ist es, einen ersten Einblick in die möglichen Trends in den Reaktionen der Hochschulen darzustellen und die Vielfalt der verschiedenen Maßnahmen und Lockerungen zu präsentieren.

Die Rückmeldungen der folgenden 8 Hochschulen werden im vorliegenden Beitrag dargestellt. Eine weitere Einrichtung wollte anonym bleiben:

- Fachhochschule Potsdam

- Humboldt-Universität zu Berlin

- Hochschule Hannover

- Hochschule der Medien Stuttgart

- Hochschule für den öffentlichen Dienst in Bayern

- Hochschule für Technik, Wirtschaft und Kultur Leipzig

- Universität Innsbruck

- Universität Wien

- Anonyme Einrichtung

Die Mehrheit (6 von 9) hat keine Änderungen an den offiziellen Vorgaben für Praktika aufgrund der Coronakrise vorgenommen. Dennoch haben viele Hochschulen versucht, der Situation Rechnung zu tragen und den Studierenden auf verschiedene Art und Weise die Erfüllung der Praktikumsrichtlinien zu erleichtern.

Wenn aufgrund der erhöhten Sicherheitsmaßnahmen kein klassisches Praktikum vor Ort möglich war, wurden hybride oder komplett virtuelle Praktika von den meisten Hochschulen zur Gänze anerkannt. Am ehesten wurden Lockerungen der erforderlichen Praktikumsdauer vorgenommen. Vier Hochschulen haben den erforderlichen zeitlichen Umfang der Praktika eingeschränkt. Dies wurde entweder für ganze Studiengänge oder auf individueller Basis in begründeten Einzelfällen entschieden. Drei der befragten Einrichtungen haben den Studierenden die Möglichkeit gegeben, fest geplante Praxisphasen im Studium auf einen späteren Zeitpunkt zu verschieben. Als enttäuschend wurde von drei Hochschulen die notwendige Streichung von geplanten Auslandspraktika empfunden. Diesbezüglich wurden einzelne Beispiele hervorgehoben, wo es kreative Lösungen für virtuelle Praktika an ausländischen Einrichtungen gab. Eine Einrichtung gab in der Befragung an, schriftliche Ausarbeitungen eines praxis-relevanten 
Themas als Ersatzleistung für Praktika anzubieten. Andere teilnehmende Einrichtungen haben diese Option nicht erwähnt.

Die inhaltlichen Vorgaben haben sich i. d. R. nicht geändert. Durch die rein virtuelle Mitarbeit wurden allerdings manche Bereiche beeinflusst. In dem Zusammenhang gab eine Einrichtung an, dass Studierende zum Ausgleich der beschränkten Möglichkeiten, Erfahrung im Veranstaltungsmanagement zu sammeln, ihre Kompetenzen in anderen eher digitalen Bereichen wie Social Media verstärken konnten.

Bibliotheken und andere Praktikumseinrichtungen wurden von zwei Hochschulen explizit für deren Flexibilität und Zuvorkommen den Studierenden gegenüber gelobt, während zwei weitere Hochschulen die häufigen Absagen von Praktikumsplätzen durch Bibliotheken bedauerten. Um die Trends in den Reaktionen von Bibliotheken auszuarbeiten, wäre eine gezielte Befragung von Bibliotheken notwendig. Eine befragte Person hat die Situation so beschrieben: „Alle lernen jetzt in der Praxis, mit einer Krise umgehen ... vielleicht verzichtbar, vielleicht sehr wertvoll.“

Die Rückmeldungen der Lehr- und Studiengänge deuten an, dass Studierende der Bibliothekswissenschaft auch in der derzeitigen Pandemie Praktika zu absolvieren haben. Auch im eventuell verkürzten Umfang haben die Studierenden daher Bedarf an geeigneten Praktikumsplätzen.

\section{Virtuelle Praktika in Bibliotheken}

Praktika in Bibliotheken bilden traditionell einen Teil der bibliothekarischen Ausbildung im D-A-CH-Bereich ab, der „als Vorbereitung auf den beruflichen Einstieg [als] unverzichtbar" betrachtet wird (Hörzer et al., 2020, S. 118). Laut der letzten Verbleibstudie von bibliothekswissenschaftlichen Absolvent* innen der Humboldt-Universität zu Berlin waren u. a. Kontakte aus einem Praktikum bei der späteren Jobsuche bei 7 \% der Befragten von Bedeutung (Kraus, 2020, S. 79).

Die Idee eines Online-Praktikums ist im Bibliothekswesen keineswegs neu. Vor allem in Bezug auf überwiegend digitale Aufgaben wie das Forschungsdatenmanagement, die aus dem Homeoffice gut möglich sind, kann ein virtuelles Praktikum zum Einsatz kommen (Franks \& Oliver, 2012, S. 274). Das virtuelle Praktikum als Format wird auch von Bird et al. hervorgehoben, da es sich zu der Erfüllung des Anspruchs auf Internationalität des vier I-Konzeptes („Intentional, Interconnected, Interdisciplinary and International") für moderne Bibliothekspraktika eignet (2011, 2015). Im Vergleich mit Praktika vor Ort kann ein flexibleres Online-Praktikum auch Stu- 
dierenden, die berufstätig sind, oder familiäre Pflichten haben, die Teilnahme ermöglichen (Franks \& Oliver, 2012, S. 275). Als besonderer Vorteil von Online-Praktika geben Franks und Oliver die Erwerbung von Kompetenzen wie kritisches Denken und in der Nutzung aktueller IT-Lösungen an (2012, S. 275). Westbrook bringt dazu Folgendes zum Ausdruck: „Remote internship programs [...] are a key step toward a tech savy librarian workforce" (2012, S. 14). Die Idee ist, dass durch den gezielten Einsatz von aktuellen IT-Tools - beispielsweise zum kollaborativen Arbeiten - zur Absolvierung eines virtuellen Praktikums die Kompetenzen der Studierenden in diesem Bereich vertieft werden. Ein rein virtuelles Praktikum kann darüber hinaus als eine Maßnahme für Nachhaltigkeit verstanden werden (Williams \& Koester, 2015, S. 377). Trotz der genannten Vorteile eines Online-Praktikums sind in der Literatur nur begrenzt Fallbeispiele zu finden (Bird et al., 2015, S. 304). Die Anzahl an Angeboten von rein virtuellen Praktika scheint aufgrund der jetzigen Lage zu wachsen ${ }^{1}$.

Die Untersuchungen der möglichen Auswirkungen der COVID-19-Pandemie auf die Angebote an virtuellen Praktika in Bibliotheken stehen noch aus. Nichtsdestotrotz gibt es erste Berichte von Praktikant*innen, die ein rein virtuelles Praktikum in den letzten Monaten absolviert haben. Dabei wird z. B. von einer Praktikantin an der Library of Congress die Flexibilität von Online-Praktika als Vorteil gesehen (McKenna, 2020). McKenna hebt in ihrem Blogbeitrag auch die einzigartige Möglichkeit hervor, durch das Format eines virtuellen Praktikums, die Arbeit in einer prestigeträchtigen Einrichtung wie der LoC kennenzulernen (2020). Wenn die notwendige Infrastruktur Praktikant*innen zur Verfügung steht, bietet ein Online-Praktikum unabhängig von der Pandemie die Option eines Auslandspraktikums auch für diejenigen, für die aus beruflichen, familiären oder finanziellen Gründen ein solches Praktikum nicht in Frage käme (Bird et al., 2015, S. 305).

\section{Online-Praktikum bei PHAIDRA}

Die Abteilung Repositorienmanagement PHAIDRA-Services freut sich dank des vielfältigen zukunftsorientierten Tätigkeitsfeldes jedes Jahr über eine hohe Anzahl an Praktikumsinteressent*innen. Im Jahr 2019 haben sechs Praktikant*innen von dem Grundlehrgang Library and Information Studies der Universität Wien, sowie sechs Erasmus-Studierende aus dem Ausland die Arbeit bei Phaidra kennengelernt.

Neben den Repositorien Phaidra und Unidam, sowie Beratungs- und Schulungsangeboten im Bereich Forschungsdatenmanagement, beteiligt 
sich die Abteilung aktiv an zwei Projekten - NewsEye ${ }^{2}$ und FAIR Data Austria ${ }^{3}$.

Um auch während der Pandemie und der damit verbundenen Unsicherheit Praktika anbieten zu können, wurde im Sommer 2020 ein neues Konzept für Online-Praktika entwickelt. Das virtuelle Praktikum ist überwiegend im Projekt FAIR Data Austria angesiedelt.

\subsection{Aufbau des Praktikums}

Ähnlich zum bestehenden Konzept für Praktika vor Ort, wird das OnlinePraktikum in zwei Bereiche aufgeteilt. Es handelt sich hierbei um einen eher theoretischen Teil, wo die Studierenden Einblicke in die verschiedenen Tätigkeitsbereiche der Abteilung gewinnen und einen praktischen Teil, in dem sie selbständig Aufgaben erarbeiten. Die Vorstellung der Bereiche, sowie die Aufteilung der Aufgaben findet in sechs Video-Konferenzen statt (jeweils 1-2 Stunden). Die restlichen 70 Stunden verbringen die Praktikant*innen bei der Erarbeitung der Aufgaben.

Die Praktikant*innen erhalten Einführungen in die folgenden Bereiche:

- Forschungsdatenmanagement und -angebote der Universität Wien

- Projekt FAIR Data Austria

- Repositorium PHAIDRA

- Repositorium AUSSDA

- Repositorium UNIDAM

- Projekt NewsEye mit Fokus auf das Tool Transkribus ${ }^{4}$

Für die Online-Treffen wird das Tool BigBlueButton verwendet, das neben den klassischen Videokonferenz-Features auch die Erarbeitung gemeinsamer Notizen ermöglicht. Dies kann für Diskussionen hilfreich sein. Die einzelnen Termine sind für 1-2 Stunden konzipiert und werden flexibel während der Praktikumslaufzeit eingeplant. Darüber hinaus kann ein Bereich in dem Cloud-System u:cloud ${ }^{5}$ der Universität Wien verwendet werden, um wichtige Dokumente wie Präsentationsfolien abzulegen und das kollaborative Arbeiten zu ermöglichen.

Da sich der Aufbau des virtuellen Praktikums im Wesentlichen von Praktika vor Ort nicht unterscheidet, war die Entwicklung des Konzeptes mit keinem erheblichen Mehraufwand seitens der Abteilung verbunden. Der Hauptunterschied lag in der Auswahl von geeigneten digitalen Tools, wofür mehr Zeit in der Vorbereitungsphase besonders bei der Erstdurchführung eingeplant werden musste. Weiters wurde über einen längeren Zeitraum (2-3 Monate) eine Liste an möglichen Aufgaben gesammelt. Die Praktikant*innen hatten 
ebenso die Möglichkeit, Themen für die Aufgaben selber vorzuschlagen und dadurch Schwerpunkte für das Praktikum zu setzen. Es wurde auch versucht, anhand der Vorerfahrungen und Kenntnisse passende Aufgaben zu finden, von denen beide Seiten am besten profitieren könnten.

Während vor Ort in der Abteilung Phaidra max. zwei Praktikant*innen gleichzeitig betreut werden können, hat ein Online-Praktikum den Vorteil, sich mehreren Studierenden gleichzeitig widmen zu können. Um eine hohe Betreuungsqualität zu gewährleisten, wurde die Teilnahme auf vier Personen begrenzt. Um den Betreuungsaufwand möglichst gering zu halten, sowie den Praktikant*innen die Möglichkeit zu geben, sich auch untereinander auszutauschen und gemeinsam an Aufgaben zu arbeiten, werden mehrere Praktika zeitlich gebündelt. Dadurch absolvieren mehrere Studierende die Praktika zeitgleich, was initial mit einem höheren Koordinationsaufwand verbunden sein könnte, im längeren Verlauf aber mit weniger Betreuungsaufwand einhergeht.

Da es eventuell seitens der Praktikant*innen Unsicherheiten bezüglich des neuen Formats geben kann, muss mit mehr Zeitaufwand für etwaige Anfragen per Mail/telefonisch oder in kürzen Video-Calls vor dem Praktikumsanfang gerechnet werden.

\subsection{Die erste Durchführung: die Versuchskaninchen}

Die erste Kohorte von vier Praktikant* innen hat vom 19.10. bis 13.11.2020 ein Online-Praktikum an der UB Wien absolviert. Es handelte sich hierbei um Studierende des weiterbildenden Masterstudiengangs der HumboldtUniversität zu Berlin im zweiten Studienjahr. Sie haben an der UB Wien ein vierwöchiges Praktikum in Teilzeit (20 Stunden/Woche) absolviert.

\section{Anforderungen der Humboldt-Universität zu Berlin}

Im weiterbildenden Masterstudiengang Bibliotheks- und Informationswissenschaft der Humboldt-Universität zu Berlin werden 18 Wochen an Praktika während oder vor dem Studium vorausgesetzt (Institut für Bibliotheks- und Informationswissenschaft, 2014, S. 2). Die Praktikant*innen haben demnach im Praktikum bei Phaidra 11\% des geforderten Umfangs absolviert.

\section{Aufgaben}

Neben der Teilnahme an den sechs Online-Terminen, haben die Praktikant*innen nach der Einführung die Aufgabe bekommen, sich mit 
den Informationen auf der Homepage ${ }^{6}$ der Abteilung und dem Repositorium ${ }^{7}$ vertraut zu machen. Anschließend konnten sie in der zweiten Praktikumswoche ihre Fragen zum Umgang mit dem Repositorium beim OnlineTreffen zum Thema Phaidra stellen. Der größte Teil der Zeit wurde mit der Ausarbeitung von 1-2 konkreten Aufgaben verbracht.

\section{Auflistung der Aufgabenbereiche}

1. Usability Testing für Repositorien

- Recherche relevanter Literatur und Erstellung einer Literaturliste

- Zusammenfassung der Möglichkeiten inklusive der Vor- und Nachteile der verschiedenen Methoden

2. Barrierefreie PDFs

- Recherche der aktuellen Best Practices

- Erstellung einer einfachen Checkliste mit den wichtigsten Aspekten

3. E-Learning-Kurse zum Thema Forschungsdatenmanagement

- Analysen von E-Learning-Kursen von anderen Einrichtungen, auch international

- Erarbeitung der Vor- und Nachteile der einzelnen Kurse/Formate

4. Bibliotheksservices für Digital Humanities

- Recherche und Zusammenfassung von Services für Digital Humanities, die von Bibliotheken in Österreich und international bereits angeboten werden

5. Analyse von Stellenausschreibungen für Data Stewards

- Recherche von aktuellen Studien, Erstellung einer Literaturliste

- Recherche von Stellenausschreibungen international (auch mehrsprachig)

- Analyse und Erstellung einer Übersicht der Stellenausschreibungen

Am letzten Praktikumstag haben die Praktikant*innen bei dem Abschlussgespräch ihre Ergebnisse in der Gruppe präsentiert.

\section{Feedback der Teilnehmenden}

Am Ende des Praktikums wurde eine Feedbackrunde organisiert. Die Rückmeldungen der Teilnehmenden waren überwiegend positiv. Sie haben angegeben, dass vor dem Praktikumsanfang Unsicherheit hinsichtlich des neuen Formats herrschte. Sie waren aber von den Inhalten, sowie dem Ablauf positiv überrascht. Die Praktikant*innen beklagten die Schwierigkeit der Praktikumssuche in der derzeitigen Pandemie. Daher haben sie das An- 
gebot, ein Praktikum wenigstens virtuell absolvieren zu können, begrüßt. Als besonders positiv wurden die folgenden Punkte erwähnt:

\section{Positiv}

- Die Betreuung und die gute Erreichbarkeit von Ansprechpersonen während der Praktikumslaufzeit

- Die Flexibilität und das Vertrauen bei der selbständigen Erarbeitung von thematisch spannenden Aufgaben

- Die Vielfalt der Bereiche, in denen sie Einführung bekommen haben

- Die entspannte Atmosphäre und Umgang auf Augenhöhe

- Möglichkeit, Themen zu diskutieren und Fragen zu stellen

Um das Angebot weiter zu optimieren, wurde auch nach Verbesserungsvorschlägen der Praktikant*innen gefragt:

\section{Verbesserungswürdig}

- Der zeitliche Rahmen für die virtuellen Einführungsveranstaltungen war zu knapp bemessen. Mehr Zeit für Fragen und Diskussion wäre wünschenswert.

- Die notwendige Technik wie Video-Konferenz-Tools oder Mikrofone haben nicht immer einwandfrei funktioniert. Es sollte nach Zeiten gesucht werden, wo der Datenverkehr online nicht so stark ist, um die Übertragung zu verbessern.

- Es fehlten weitere regelmäßige informelle Treffen ohne Agenda, um sich zu aktuellen Fragen und über den Stand der Aufgaben austauschen zu können.

- Die Bibliothek als Ort ist sehr beliebt und eine Führung durch die Räumlichkeiten bildet i. d. R. einen Teil von Praktika vor Ort ab. Ein gemeinsamer virtueller Durchgang durch die Bibliothek wäre wünschenswert.

\section{Lessons Learned}

Sowohl die Erfahrungsberichte aus der Abteilung Repositorienmanagement PHAIDRA-Services der UB Wien als auch die Rückmeldungen der ersten virtuellen Praktikant*innen lassen auf einen positiven Eindruck mit dem Format schließen. Weiters können folgende Lessons Learned aus den 
Verbesserungsvorschlägen und Überlegungen der Betreuer*innen und Praktikant*innen abgeleitet werden, die anderen Einrichtungen Hinweise für die Planung eigener Online-Praktika geben könnten.

Kommunikation vor und während des Praktikums ist für ein virtuelles Praktikum entscheidend. Es ist sinnvoll, dass eine Person aus der Abteilung als konkrete Ansprechperson während der Praktikumslaufzeit den Praktikant*innen hilfsbereit zur Seite steht und Fragen zeitnah per E-Mail oder telefonisch beantwortet. Auch das aktive Zugehen auf Praktikant*innen, um möglichen Schwierigkeiten, Bedürfnissen oder offenen Fragen zu begegnen, hat sich als effektiv bewiesen.

Ähnlich wie bei einem klassischen Praktikum vor Ort wird Vielfalt der vermittelten Inhalte als positiv betrachtet. Einerseits ist durch die Aufteilung der Betreuung auf mehrere Kolleg*innen der individuelle Zeitaufwand geringer, andererseits gewinnen die Praktikant*innen einen besseren Überblick über die Arbeit einer Abteilung/Bibliothek.

Eine konkrete interessante Aufgabe, die die Praktikant*innen selbständig und flexibel erarbeiten können, ist für beide Seiten wesentlich für den Erfolg.

Obwohl sowohl die Mitarbeiter*innen als auch die Praktikant*innen auf technische Schwierigkeiten (vor allem bezüglich der Übertragung bei Video-Konferenzen) als Hürde bei dem Praktikum hingewiesen haben, haben Bibliotheken i. d. R. wenig Einfluss darauf. Die Idee, nach eventuell weniger frequentierten Zeiten im Netz zu suchen, erscheint hierbei als sinnvoll. Ebenfalls hat sich das gründliche Testen der Funktionalitäten von verschiedenen Video-Konferenz-Tools im Voraus als zielführend bei der Entdeckung von möglichen Problembereichen erwiesen.

Der informelle Austausch hat beiden Seiten, den Mitarbeiter*innen und Praktikant*innen, während des virtuellen Praktikums gefehlt. Demnach könnten mehrere gezielt eingeplante regelmäßige Online-Treffen ohne Agenda dabei helfen, Praktikant*innen willkommen zu heißen, sich in der Gruppe auszutauschen und auf mögliche Fragen einzugehen. Ebenso könnten kurze Einzelgespräche zusätzlich zu den Gruppen-Calls für den (nicht ausschließlich) fachlichen Austausch nützlich sein. Auch das Angebot seitens der Bibliotheksmitarbeiter*innen im weiteren Austausch über die Praktikumslaufzeit hinaus zu bleiben, z. B. bezüglich möglicher Abschlussarbeitsthemen, kann bei Praktikant*innen auf Resonanz stoßen.

Während der Praktikumslaufzeit der ersten Gruppe hat die Universitätsbibliothek Wien einen virtuellen Rundgang ${ }^{8}$ durch die Bibliotheksräumlichkeiten veröffentlicht und ein Link wurde an die Teilnehmenden weitergeleitet. Aus dem Feedback der Praktikant*innen geht hervor, dass 
eine gemeinsame Online-Führung durch die Bibliothek (z. B. mithilfe eines virtuellen Rundgang-Tools) erwünscht wäre. Dies könnte ein Weg sein, eine traditionelle Bibliotheksführung als Teil eines Praktikums auch virtuell anzubieten.

Aufgrund der positiven Erfahrung mit dem neuen Format haben die Mitarbeiter*innen der Abteilung Phaidra beschlossen, Online-Praktika auch über die Krisenzeit hinaus anzubieten, um vor allem einen internationalen Austausch mit Praktikant*innen aus dem Ausland, wie in der Studie von Bird et al. (2015) beschrieben, zu ermöglichen. Bei Praktikant*innen, die nach Wien kommen können, könnte ein hybrides Modell angeboten werden. Dies würde bei nicht ausreichenden Arbeitsplätzen die Betreuung von mehreren Praktikant*innen auch vor Ort ermöglichen. Virtuelle Praktika können auch zur Erhöhung der Sichtbarkeit der eigenen Arbeit und Projekte beitragen und als Marketingmaßnahme eingesetzt werden.

Es wurden seit der ersten Durchführung des virtuellen Praktikums mehrere Studierende in kürzeren Online-Praktika (3-5 Tage) in der Abteilung Phaidra betreut. Hierbei fand das im vorliegenden Beitrag beschriebene Konzept in einer verkürzten Form Anwendung. Das Konzept der virtuellen Praktika wird anhand des Feedbacks und der Erfahrungen in der Abteilung in den nächsten Monaten weiter ausgebaut, um es in Zukunft auch international bewerben zu können.

\section{Zusammenfassung}

Im November 2020 wurde eine Umfrage an deutschsprachigen Hochschulen, die bibliothekarische Ausbildung anbieten, durchgeführt. Die Ergebnisse zeigten, dass sich Praktikumsvorgaben in den Zeiten der Corona-Pandemie im Wesentlichen nicht geändert haben. Die Studierenden müssen i. d. R. weiterhin unter häufig erschwerten Bedingungen nach Praktikumsplätzen suchen. Die Abteilung Repositorienmanagement PHAIDRA-Services ist diesem Problem begegnet, indem in der Abteilung ein Online-Praktikumsprogramm erstmalig eingeführt wurde. Dabei wurden vor allem die Aspekte der zeitlichen Flexibilität bei der Erarbeitung von Aufgaben und die Möglichkeit eines internationalen Austausches als positiv bewertet. Bei der Weiterentwicklung des Angebots sollten neben den Vorträgen zu verschiedenen Themen auch mehrere informelle Treffen mit den Teilnehmenden eingeplant werden. Darüber hinaus ist eine kontinuierliche Überprüfung der technischen Möglichkeiten von verschiedenen Tools von Relevanz. 
Online-Praktika stellen auch über die derzeitige Krisensituation hinaus ein spannendes Format dar, das Studierenden der Bibliotheks- und Informationswissenschaft praktische Erfahrung in Bibliotheken ermöglichen kann. Bibliotheken haben dadurch die Möglichkeit, neue Ideen und Perspektiven für ihre Arbeit im digitalen Zeitalter zu gewinnen. Insbesondere im Hinblick dessen, dass Online-Praktika ohne große finanzielle Hindernisse wahrgenommen werden können, um auch einen interkulturellen Austausch über Grenzen hinweg zu fördern.

Tereza Kalová, MA (Res), MA LIS ORCID iD: https://orcid.org/0000-0002-1764-7228 Universität Wien, Universitätsbibliothek E-Mail: tereza.kalova@univie.ac.at

\section{Literatur}

Bird, N. J., Chu, C. M., \& Oguz, F. (2011). Four Is of Internships for the New Information Society: Intentional, Interconnected, Interdisciplinary and International. Proceedings of the World Library and Information Congress: 77th IFLA General Conference and Assembly. https://www. ifla.org/past-wlic/2011/120-bird-en.pdf

Bird, N. J., Chu, C. M., \& Oguz, F. (2015). Internship in LIS education: An international perspective on experiential learning. IFLA Journal, 41(4), 298-307. https://doi.org/10.1177/0340035215596352

Franks, P. C., \& Oliver, G. C. (2012). Experiential learning and international collaboration opportunities: Virtual internships. Library Review, 61(4), 272-285. https://doi.org/10.1108/00242531211267572

Hörzer, B., Pum, G., Rezniczek, A., \& Schneider-Jakob, M. (2020). Neues aus der bibliothekarischen Aus- und Fortbildung in den Studienjahren 2018/19 und 2019/20. Mitteilungen der Vereinigung Österreichischer Bibliothekarinnen und Bibliothekare, 73(1), 118-126. https://doi. org/10.31263/voebm.v73i1.3414

Institut für Bibliotheks- und Informationswissenschaft. (2014). Richtlinie für die berufspraktische Tätigkeit im weiterbildenden Masterstudiengang „Bibliotheks- und Informationswissenschaft" im Fernstudium. https://www.ibi.hu-berlin.de/de/studium/studiengaenge/fernstudium/ ordnungen/praktikum/praktikumsrichtlinie-2014/at_download/file

Kraus, E. (2020). „Quo vadis, IBI-Fernstudent*in?“. Masterarbeit, Humboldt-Universität zu Berlin. https://doi.org/10.18452/21052 
McKenna, S. (2020, Juni 22). A New Sense of Normal: Libraries and Virtual Internships. Hack Library School. https://hacklibraryschool. com/2020/06/22/a-new-sense-of-normal-libraries-and-virtualinternships/

Westbrook, R. (2012). How to Use Free Online Tools to Recruit and Manage Remote LIS Interns. Library and Leadership Management, 26(3/4). https://doi.org/10.5860/llm.v26i3/4.6313

Williams, B. F., \& Koester, A. (2015). No money, no travel, no problem. In: Hauke, P., Latimer, K., \& Werner, K. U. (eds.). The Green Library - Die grüne Bibliothek. Berlin, Boston: De Gruyter Saur, 373-380. https://doi.org/10.1515/9783110309720.373 (Open Access verfügbar unter: https://doi.org/10.18452/2316)

* Alle Websites und Links wurden zuletzt am 28. Dezember 2020 aufgerufen.

1 Siehe z.B. die Auflistung der Praktikumsangebote der UCLA: https:// is.gseis.ucla.edu/programs/mlis-degree/mlis-internships/internshiplisting-2020/

2 https://www.newseye.eu/

3 https://forschungsdaten.at/fda/

4 https://readcoop.eu/transkribus/

5 https://ucloud.univie.ac.at/

6 https://datamanagement.univie.ac.at/

7 https://phaidra.univie.ac.at/

8 https://bibliothek.univie.ac.at/virtueller_rundgang.html 